Daniel Salas, Sistema Recomendador

\title{
DISEÑO E IMPLEMENTACIÓN DE UN SISTEMA RECOMENDADOR PARA APOYAR LA SELECCIÓN DE PROGRAMAS ACADÉMICOS EN EDUCACIÓN SUPERIOR
}

\section{DESIGN AND IMPLEMENTATION OF A RECOMMENDER SYSTEM FOR SUPPORTING THE SELECTION OF ACADEMIC PROGRAMS IN HIGHSCHOOL}

\author{
Daniel J. Salas ${ }^{1}$, Carlos J. Urango ${ }^{2}$, Darío Velásquez ${ }^{3}$, Julio Martínez ${ }^{4}$ \\ Recibido para publicación: 18 de julio de 2012 - Aceptado para publicación: 15 Diciembre de 2012
}

\section{RESUMEN}

En la cotidianidad hay que enfrentarse a la toma de decisiones, unas más importantes que otras pero sin importar cuan trascendentes sean, representan un inconveniente para quien se tiene que decidir entre un grupo de opciones, pues muchas veces surge la confusión mental y las cuestiones sobre porque no se escogió tal opción en vez de otra. Cada día son más los individuos que recurren a las opiniones de terceros para tomar decisiones que le signifiquen un mayor provecho, ya que al desconocimiento sobre las opciones a elegir, tiende a dificultar la escogencia. Gracias a hechos como el expuesto la sociedad ha forzado la creación de entidades automáticas o semiautomáticas (de aquí en adelante Sistemas Recomendadores) que ejecuten el filtrado o selección de información relevante para los individuos interesados en un grupo de opciones, con lo cual se facilita la toma de decisiones; es por ello, que los Sistemas recomendadores se usan en una gran variedad de ámbitos como el comercio electrónico, la medicina y en un campo que siente los beneficios de herramientas como esta: la educación.

Este proyecto se ubica en la línea de los sistemas recomendadores ya que está desarrollado para facilitar la toma de decisiones de los estudiantes que cursen décimo y undécimo de educación básica secundaria, teniendo en cuenta sus conocimientos en y sus preferencias. Este sistema soportado en la web, fue construido teniendo en cuenta el modelo tricapa (lógica, presentación y persistencia) usando el paradigma de orientación a objetos, el lenguaje de programación JAVA y su extensión hacia plataforma web, JSP. Adicionalmente el sistema cuenta con una serie de características que le

\footnotetext{
1 Ingeniero Sistemas, MSc. Docente, Universidad de Córdoba, Montería - Colombia danielsalas@correo.unicordoba.edu.co

2 Ingeniero de Sistemas, Universidad de Córdoba, Montería - Colombia cajaur_2024@hotmail.com

3 Ingeniero de Sistemas, Universidad de Córdoba, Montería - Colombia

${ }^{4}$ Ingeniero de Sistemas, Universidad de Córdoba, Montería - Colombia jcmarcher@gmail.com
} 
Daniel Salas, Sistema Recomendador

permiten la administración de los datos usados en el proceso de recomendación así como también la elaboración de consultas y reportes.

PALABRAS CLAVES: Sistemas Recomendadores, educación, comercio electrónico, orientación vocacional, información.

\section{ABSTRACT}

In everyday life we must face the decision-making, a more important than others but no matter how transcendent, represent a disadvantage for those who have to decide between a group of options, because often there is confusion and questions about mental because this option was chosen over another. More and more individuals turn to the opinions of others to make decisions that will mean more profit, since the lack of options to choose from, tends to make the choice. Thanks to events like the above society has forced the creation of entities or semi-automatic (here in after recommender systems) implementing the filtering or selection of relevant information for individuals interested in an option group, which facilitates the decision making, which is why the recommended systems are used in a variety of areas including electronic commerce, medicine, and in a field that is feeling the benefits of tools like this: education.

This project is located in the line of recommender systems as it is developed to facilitate decision making of students attending the tenth and eleventh of basic secondary education, taking into account their knowledge and preferences. This web-supported system was built taking into account

the three-layer model (logic, presentation and persistence) using the paradigm of object oriented programming language Java and its extension to web platform, JSP. Additionally, the system has a number of features that allow the administration of the data used in the referral process as well as querying and reporting.

KEYWORDS: recommender system, education, electronic commerce, vocational guidance, information.

\section{INTRODUCCIÓN}

Un programa académico de educación superior, suele convertirse en el objetivo de cualquier estudiante que está finalizando su ciclo escolar. En este proceso de cambio el estudiante se enfrenta a la cuestión inevitable sobre qué programa académico de educación superior se debe elegir para estudiar. Sin embargo la decisión por parte del estudiante generalmente es tomada sin tener en cuenta ningún tipo de asesoría o instrucción, es decir, se basan únicamente en cualquier información percibida en el medio o en suposiciones personales acerca del programa a estudiar, dejando a un lado factores muy importantes como lo son los fundamentos académicos en los que se centra cada programa, así como los intereses y habilidades que debe tener el aspirante y lo que este deberá saber y saber hacer en un futuro. Este hecho ocasiona que se tome una decisión muy poco acertada, ya que el estudiante no se percató de que no correspondía con el perfil definido en el programa escogido, ni contaba con la suficiente preparación académica, la cual debió haber adquirido en la escuela, por lo tanto el nivel académico no es el suficiente para lograr el desempeño requerido para completar con éxito el programa de pregrado. Para aprovechar la utilidad de los sistemas recomendadores se tomó la idea de crear un sistema de información que recomiende programas de pregrado a los estudiantes que aspiren ingresar a la educación superior, teniendo en cuenta sus necesidades e intereses. Para definir las entradas del sistema, hay que tener en cuenta que cada programa académico tiene definido en qué áreas del conocimiento enfatizan su plan de estudios, el perfil, vocación, habilidades e intereses que son necesarios en cualquier postulante; Además de esto se conocen otros aspectos relevantes como los resultados de las pruebas ICFES (Instituto Colombiano de Fomento de la Educación Superior), las cuales 
actualmente son un requisito para el ingreso a la educación superior en Colombia. El interés principal de este trabajo se centra en demostrar que el uso de tecnologías de recomendación basadas en conocimiento influye positivamente en la toma de decisiones de los estudiantes que desean ingresar en la educación superior.

\section{ESTADO DEL ARTE}

El desarrollo de los sistemas recomendadores, se dio desde principios de la década de los 90 [1] cuando se ofreció el servicio de filtrado de noticias a grupos de usuarios. El proceso era mostrar u ofrecer noticias que serían de potencial interés para ellos. El filtrado sería la primera concepción o aproximación de lo que es una recomendación ya que se encargaba de desechar aquellas noticias que no eran tan interesantes para los grupos de usuarios. Esa situación sentó una base acerca de cómo sería el proceso de recomendación realizado por una herramienta de software.

Para hacer la transición hacia la siguiente etapa se requeriría de un refinamiento de las especificaciones que se deben tener en cuenta para desarrollar un sistema recomendador, como el manejo de información más compleja y la implementación de la retroalimentación. El primer sistema que cumplía con tales exigencias fue el Tapestry [8], desarrollado por XeroX PARC. Este sistema permitía mediante la retroalimentación proporcionada por los usuarios sobre artículos o noticias, establecer sí el contenido de un documento era importante. Inicialmente este tipo de procesos fueron denominados como filtrado colaborativo, ya que aparte de seleccionar la información pertinente de acuerdo a condiciones de filtrado dadas por los ítems valorados, lo hacían mediante los comentarios hechos a los documentos que eran recomendados; estas opiniones eran usadas para las búsquedas hechas por otros usuarios. El trabajo realizado por Housman y Kaskela [6] fue otra acertada construcción de un sistema recomendador pues informaba a científicos sobre artículos que fueran de interés para ellos, suministrando palabras claves dentro de un perfil de usuario; esas palabras eran usadas para buscar coincidencias dentro de un conjunto de documentos [6].

El campo de acción de los sistemas recomendadores a través del tiempo fue aumentando, ya no solo se ofrecían recomendaciones de documentos o artículos, progresivamente se fueron incluyendo productos comerciales como discos compactos, libros, automóviles y una variedad de ítems propuestos en el mundo de la compra y venta [1].

Los sistemas de recomendación están clasificados como entidades que recolectan opiniones sobre un conjunto de objetos relacionados en alguna forma con un grupo de usuarios [17], las opiniones captadas sirven de guía para dirigir a los usuarios a objetos de interés para ellos [3]. Por ejemplo los sistemas recomendadores, captan opiniones de usuarios, acerca de productos objetos o ítems tales como, páginas web, libros, restaurantes, etc., luego clasifican tales opiniones para finalmente utilizarlas en dos formas posibles: para sugerir nuevas páginas, restaurantes o libros, o para predecir la utilidad que tiene uno de estos objetos para un usuario en particular [2]. La contextualización de un sistema de recomendación explícitamente lleva inmerso el concepto de recomendación, que inicialmente se entiende como una sugerencia sobre algo. Más adelante se expondrá con claridad a que se refiere este concepto. Es preciso resaltar que un sistema de este tipo solo actúa como un intermediario en el proceso de recomendación. Un sistema de recomendación es aquel que predice cuan útiles o interesantes resultan ciertos ítems para un usuario dado [9]. Esta definición no se limita a considerar las opiniones de otras personas como base 0 criterio de selección de ítems para luego recomendarlos, sino que de modo más amplio permite el uso de otros elementos para predecir el interés de los usuarios.

Dentro del contexto educativo resaltan los siguientes sistemas: CourseAgent [7] es un 
sistema que brinda acceso personalizado sobre los cursos que están disponibles para los estudiantes; fue desarrollado en la Escuela de Ciencias de la Información de la Universidad de Pittsburgh. Este es un sistema que usa la técnica de recomendación social y alimenta la precisión de las mismas a través de la retroalimentación explícita, la cual se efectúa mediante la valoración explicita de los cursos otorgándoles un grado de relevancia, calificándolos de acuerdo a la carga de trabajo propia de cada curso y la evaluación final sobre un curso que ya fue desarrollado.

La Universidad de Stanford desarrolló el sistema CourseRank [12] el cual ayuda a los estudiantes a mejorar la toma de decisiones respecto a la planeación de un programa académico que resulte satisfactorio. Es decir un programa académico resulta satisfactorio cuando los estudiantes al finalizar los cursos tomados obtienen el mayor rendimiento académico posible.

Si bien la clasificación de los SR no es única se pueden identificar dos grandes grupos de recomendadores [13], dicha clasificación se hace teniendo en cuenta el conocimiento usado para realizar las recomendaciones: los Basados en contenido [11], son aquellos que realizan la recomendación apoyándose en la descripción dada a los ítems, y los Colaborativos [10], que utilizan valoraciones asociadas a los productos dadas por el o los usuarios y realizar recomendaciones basadas en gustos similares.

Sin embargo, al transcurrir del tiempo otras técnicas han sido propuestas para desarrollar recomendadores, actualmente se consideran las Basadas en conocimiento [4] usan el conocimiento para inferir que ítems o conjunto de ellos cubren o satisfacen las necesidades de un usuario [14]; los Demográficos los cuales usan afinidades entre datos demográficos de los usuarios como la educación, su ocupación o la edad, con los cuales se generan clases a las que se les podrá inferir que tipo de ítems pueden equipararse a esa información demográfica y efectuar la recomendación a partir de ellas [15].
Las técnicas mencionadas tienen sus limitantes, para eliminar dichas limitaciones aparecieron los recomendadores híbridos [3]. Este tipo de sistemas combinan diferentes técnicas o paradigmas de recomendación para eliminar dos situaciones problemáticas que se dan al usar otro tipo de método individualmente: la primera es para eliminar o atenuar los problemas de un tipo de sistema concreto y la segunda para disminuir los efectos de la poca precisión de las recomendaciones.

\section{MATERIALES Y MÉTODOS}

El proceso de la investigación se realizó en las siguientes fases.

\subsection{Planeación.}

El desarrollo del Sistema Recomendador queda reflejado en un documento en donde se especifican cada una de las siguientes etapas:

- Estudio de la Factibilidad.

- Identificación de las Tareas del Sistema Recomendador

- Capacidad Funcional Inicial.

\subsection{Establecimiento de las Conductas de Entrada.}

Se definen las conductas de entrada del sistema. Estas deben ser escogidas para cumplir con la exigencia de que satisfagan la correcta selección de datos de un sistema recomendador basado en conocimiento.

- Identificación y Selección de las conductas de entrada.

- Extracción de características relevantes para la construcción del test.

- Formulación de un test basado en las características de cada programa.

- Especificar la proporción que tendrán las conductas de entradas que serán utilizadas para efectuar los cálculos correspondientes en el proceso de recomendación.

\subsection{Modelado del Requerimiento de Administración y Reportes del Sistema.}

Se desarrolló el modelado de un módulo de administración que permita gestionar los datos 
de los usuarios, los programas, las características, los formularios, las preguntas del test, los tipos de respuestas, las asignaturas y las puntuaciones dadas a las conductas de entrada. La gestión se extiende a los procesos de: inserción, consulta, modificación y eliminación de registros de la información mencionada para gestión. Adicionalmente el módulo de reportes donde se puedan realizar consultas sobre las recomendaciones hechas, basadas en diversos criterios como nombre del estudiante, nombre del programa o fecha.

\subsection{Definición de las Reglas Generales de Cálculo y Explicación del Sistema.}

Se contempló el desarrollo de las fases de:

Creación de un modelo de regla general que realice los cálculos sobre todas las

características en todos los programas

(existentes y de nuevo ingreso) que permita la creación de un perfil de usuario basado en el grado de satisfacción de las características en cuestión.

Creación de un modelo de regla general que ejecute los cálculos sobre todas las asignaturas para todos los programas que permita la generación de un perfil basado en el nivel de conocimiento en las áreas evaluadas por el ICFES.

Describir el modelo de razonamiento que se utilizó para obtener la recomendación.

\subsection{Codificación y verificación.}

Durante esta fase se traducirán los diferentes modelos al lenguaje de programación seleccionado junto con las tecnologías de apoyo, para ello se ejecutaran los siguientes procesos:

Codificación del módulo de administración de la información usada durante el proceso de recomendación.

Codificación de las reglas generales de cálculo y el modelo de razonamiento usado para explicar al estudiante el porqué de la recomendación hecha.

Construcción de una interfaz gráfica de usuario que permita recolectar los datos requeridos para el establecimiento de los perfiles.
Realización de diversas pruebas preliminares para verificar que el sistema tenga un correcto desempeño, es decir que el sistema arroje resultados coherentes en situaciones muy parecidas.

\section{RESULTADOS}

\subsection{EL SISTEMA STUDENT-R}

Es un sistema de recomendación basado en conocimiento que sirve como guía en la toma de decisiones de los estudiantes de décimo y undécimo que lo utilicen, en lo que a escogencia de un programa de educación superior se refiere. Las recomendaciones se tienen en cuenta un número limitado de ítems los cuales corresponden a los programas ofrecidos en la universidad de Córdoba.

Esta es una aplicación web, que capta los intereses de los estudiantes a través de un test diseñado específicamente para tal fin. También captura los datos referentes al puntaje obtenido en cada una de las asignaturas contenidas en el núcleo común evaluadas por el ICFES. Estos datos son procesados mediante una serie de cálculos con el fin de determinar que programa satisface en mayor medida la necesidad 0 interés del usuario que lo utilice.

Las conductas de entradas del sistema son dos: un test vocacional y los puntajes de las asignaturas de las pruebas saber 11. La elección de estas entradas se debe a que en muchos casos un test vocacional indica muy acertadamente lo que un estudiante desea estudiar. Para la presente investigación el test se desarrolla en dos fases: la primera que permite buscar un perfil más general teniendo en cuenta las preferencias sobre las facultades y se selecciona aquella en donde el estudiante demuestre el mayor interés; y la segunda en gustos más específicos que ayuden a determinar en qué programa de la facultad seleccionada en la primera fase, el estudiante pueda tener un mejor desempeño. En el caso de los puntajes de la prueba saber, se escogió porque es el criterio de selección que se utiliza 
para escoger los estudiantes que ingresaran a cursar cualquier programa ofrecido en la Universidad de Córdoba.

El funcionamiento del sistema recomendador en términos generales es el siguiente: para efectuar una recomendación el estudiante que lo utilice deberá responder por lo menos el test vocacional, opcionalmente puede ingresar los resultados obtenidos en cada asignatura evaluada en la prueba Saber 11. Si sólo ingresa el test el sistema hará una serie de cálculos que encasillaran el perfil del estudiante en el programa más adecuado y le mostrará gráficamente el nivel de creencia que el sistema tiene sobre el programa recomendado de satisfacer sus intereses. Si el estudiante ingresa ambas conductas de entrada el sistema combinara los perfiles obtenidos de la captura de los datos en el test y los puntajes de la prueba Saber 11 y le mostrará al estudiante el programa recomendada solo por sus preferencias y otra carrera recomendada en donde se mezclan los intereses y el conocimiento de las diferentes asignaturas.

Todo sistema Recomendador es bien valorado de acuerdo al grado de exactitud que tengan sus recomendaciones sobre los intereses del usuario, por ello es necesario hacer énfasis en el proceso que abarca la generación de estas, por lo tanto si no se posee una idea del proceso de construcción de las salidas del sistema, se podrían llegar a tener recomendaciones que quizás nunca se ajustarán al perfil de la persona que lo esté utilizando.

Por lo tanto, para desarrollar un sistema capaz de ayudar a un estudiante de último grado de secundaria a escoger un programa de pregrado de una universidad, se hace necesario tener bien definido "cómo el sistema va a ser capaz de captar lo interesante que es para este estudiante cada uno de estos programas, y cómo dividir en fases todo el proceso de recomendación", lo que implica crear un modelo que de soporte a la consolidación de estas fases, necesarias para la elaboración de una buena recomendación, y a su vez para elevar el grado de confianza que el usuario tenga sobre esta salida que el sistema generó. A raíz de esto se construyó dicho modelo ilustrado en la figura

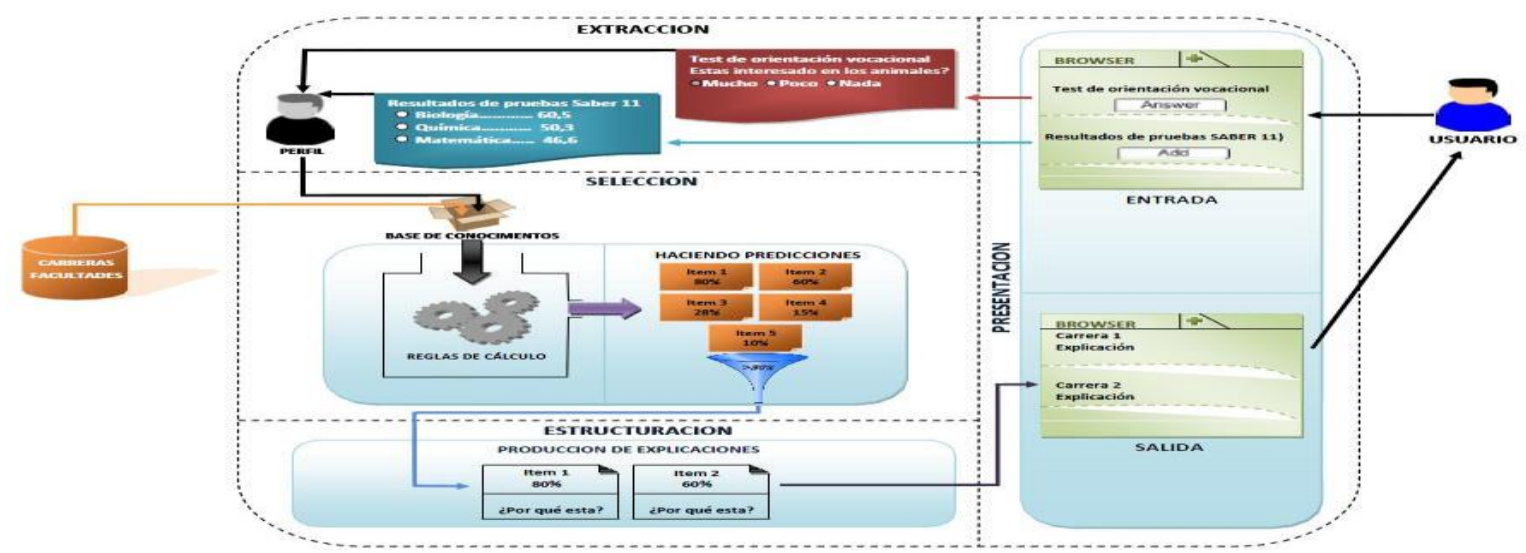

Figura 1. Modelo General Student-R

Como se puede observar, el modelo para el sistema está dividido en cuatro fases: extracción, selección, estructuración y presentación. Las tres primeras conformadas por módulos funcionales que se deben llevar a cabo para que dichas recomendaciones tengan la organización necesaria y sean certeras, convenciendo al estudiante de que los programas de pregrado recomendados son los que mejor se ajustan a su perfil.

En primer lugar, el proceso de entrada en la fase de presentación permite al estudiante realizar un test de orientación vocacional e ingresar al sistema los resultados obtenidos en su prueba de estado ICFES, con el fin de dar 
paso a la ejecución de la fase posterior llamada fase de extracción, en donde se capta información del perfil de dicho estudiante teniendo en cuenta las respuestas y resultados de dicho test vocacional y sus pruebas ICFES respectivamente.

Luego, entra en acción la fase de selección, que utiliza la información captada del mencionado perfil junto con la información de los atributos que poseen los programas de pregrado albergados en la base de datos, para generar las predicciones y escoger las que podrían ser más interesantes para él.

Más tarde, la fase de estructuración le proporciona el contenido necesario a las predicciones que pasaron el filtro de la selección anterior (se le agregan explicaciones y valoraciones), para convencerlo de que estos son los programas de pregrado más aptos a estudiar.

Al final, nuevamente se llega a la fase de presentación, más específicamente al proceso de salida, en donde se le da lugar a la parte visual de los resultados obtenidos por el sistema, mostrándole las recomendaciones al estudiante.

\subsection{DISEÑO DE LAS REGLAS GENERALES DE CÁLCULO.}

En primer lugar el software filtrará aquella facultad en donde el estudiante obtuvo el máximo puntaje. Para ello se establece que por cada facultad evaluada por el sistema, se calculen los promedios para todas y por un método de selección, elige la de mejor ranking. El promedio se calcula de la siguiente forma: como cada pregunta evalúa un aspecto en el que el valor de la respuesta varía de 0.1 a 1 siendo 1 el máximo interés y el 0.1 el mínimo; se suman los valores contestado por el estudiante (número entre 0.1 y 1 ) que pertenezcan a la misma facultad y se divide entre el número de preguntas que afectan a cada facultad. Se recorren todas las facultades y se les haya el promedio del interés del estudiante, se almacena ese valor dentro de un atributo y se elige el de mayor valor.
Para las características por programa, el sistema debe filtrar aquellos programas que pertenecen a la facultad con el mayor promedio que sería la primera regla de la segunda fase. Luego el sistema tiene dos opciones calcular únicamente sobre las preferencias o hacer un cálculo combinado de las preferencias con los puntajes ingresados de la prueba saber 11. Si es cálculo por preferencias se recorren todas las carreras filtradas y se les haya un promedio ponderado sobre todas las características, utilizando los puntajes requeridos para la característica en cuestión y el puntaje dado por el estudiante al momento de responder. Se guarda este promedio en un atributo para todos los programas. Con un método de selección se determina cual fue la carrera con mejor promedio ponderado, resultando ser la carrera que será recomendada. $\mathrm{Si}$ es cálculo combinado el sistema usara el resultado arrojado por el cálculo por preferencias y junto con el cálculo por asignaturas que se define como el promedio ponderado del puntaje de las asignaturas junto con el valor de las mismas ingresado por el estudiante. Un caso general del cálculo por asignatura es la multiplicación del puntaje ingresado por el estudiante y la puntuación de la asignatura en el programa, se divide el resultado entre la suma de las puntuaciones exigidas para la carrera. Estas puntuaciones exigidas reflejan el nivel de competencia en cada programa. Los promedios ponderados son utilizados por otro método de selección para determinar cuál programa obtuvo la mejor puntuación. Una vez hecho los cálculos de preferencias y por asignaturas, se divide el resultado por dos para dar la misma proporción o peso a las conductas de entrada.

\subsection{MÓDULO DE EXPLICACIÓN.}

Este módulo tiene como funcionalidad reforzar la confianza que tiene el usuario estudiante sobre la recomendación que se le ha hecho. Para ello se toman las características de aquella carrera que resulto recomendada y se 
construye un texto que le explique al estudiante el porqué de la recomendación brindándole argumentos que se basan en las características con mejor ranking y que tuvieron un mayor peso al momento de decidir qué carrera debía ser recomendada.

\subsection{DISEÑO E IMPLEMENTACIÓN DE LA INTERFAZ GRÁFICA.}

Al tratarse de un software en la web, para crear los elementos de captura de datos se usaron las tecnologías HTML, JSP, AJAX y Struts para el manejo dinámico del software. La interfaz gráfica del sistema se divide en tres grandes apartados. El primero el módulo administrativo en donde se gestionan los datos de las entidades que intervienen en el proceso de recomendación: facultades, programas, características, formularios, preguntas, respuestas, puntuaciones, asignaturas y usuarios. El siguiente módulo es el encargado de la captura de las preferencias de usuario a través del test de preferencias y el llenado de

los puntajes obtenidos en la prueba saber 11.

Y por último el módulo de reportes en donde se ofrecen consultas sobre las recomendaciones hechas. Las consultas se hacen dependiendo de la selección de un criterio de búsqueda, ya sea por nombres, por carreras recomendadas o por intervalos de fechas.

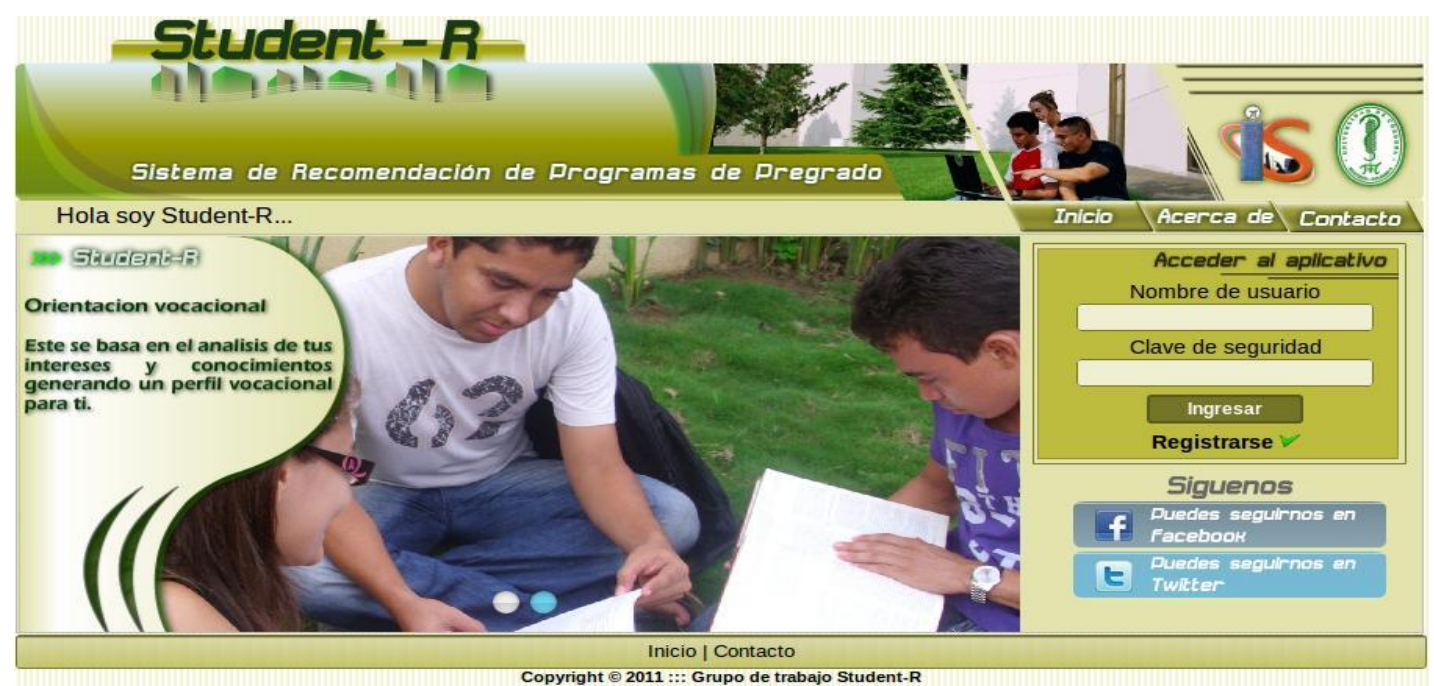

Figura 2. Interfaz de Acceso Student-R

Según las premisas y enunciados anteriores se logró construir el sistema Student-R, su interfaz de acceso se muestra en la Figura 2 Para este sistema se distinguen tres roles: administrador, estudiante y supervisor, estos roles corresponden a cada una de las secciones mencionadas. Para el primer y tercer rol se acede directamente con la clave suministrada por el creador del sistema, mientras que para el estudiante existe un formulario (Figura 3) donde puede registrarse y así poder establecer un nombre de usuario y

Sección Estudiantes: es la sección fundamental del sistema, en ella el estudiante una contraseña que le permita entrar a la sección de estudiante.

El sistema consta de tres secciones funcionales:

Sección Administrativa: en donde el administrador del sistema puede gestionar todos los datos usados en el proceso de recomendación, ya sean facultades, programas, características, asignaturas, formularios, preguntas, respuestas, puntuaciones y usuarios (Figura 4).

Adicionalmente el administrador puede editar sus datos de usuario y cambiar la contraseña. 
que se ha registrado responde las preguntas del test, y opcionalmente puede ingresar los

Sección de Reportes: aquí se pueden consultar las recomendaciones hechas a lo largo del tiempo en que el sistema ha sido usado. Usa varios criterios de búsqueda para resultados obtenidos en la prueba SABER 11.

obtener datos más detallados sobre la recomendación.

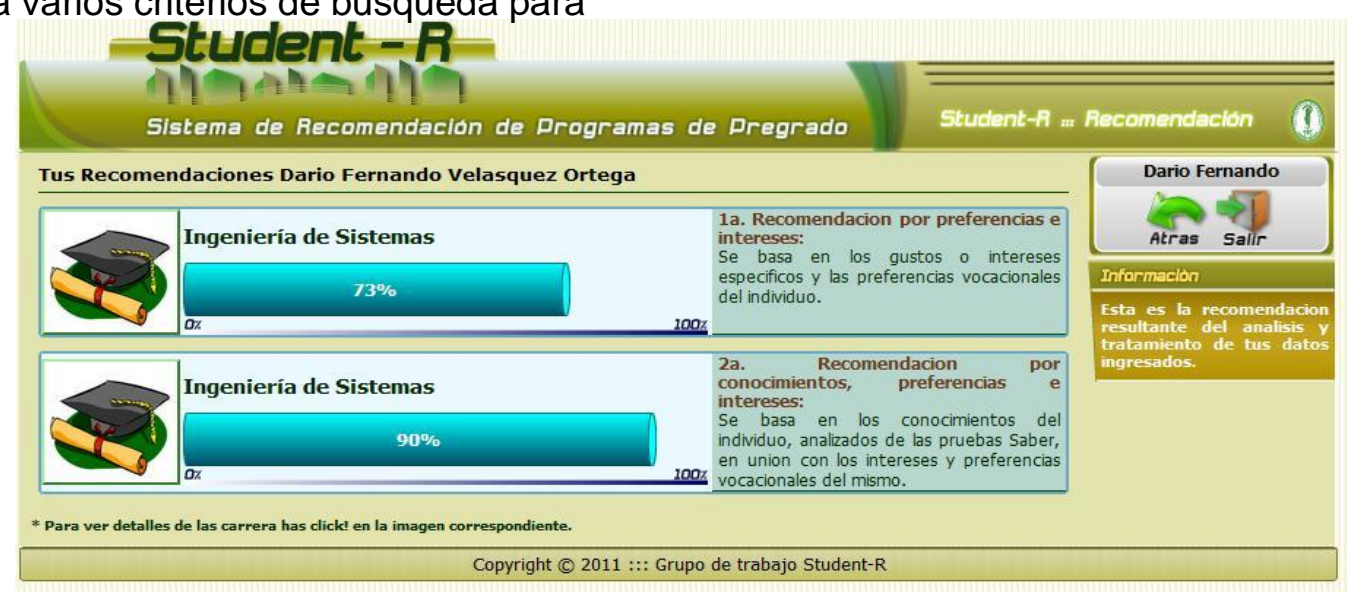

Figura 3. Recomendación Combinada.

\section{CONCLUSIONES}

Los sistemas recomendadores son una herramienta de apoyo muy importante para tomar decisiones en cualquier campo, por ende su desarrollo debe ser un proceso minucioso que defina muy bien qué tipo de información requerirá del usuario y/o qué tipo de información tendrá sobre los ítems a recomendar, para que la calidad de la recomendación satisfaga en la mayor medida posible la necesidad del usuario y se vean llenadas sus expectativas.

En el desarrollo del sistema recomendador Student- $R$ se utilizaron tecnologías asequibles y confiables en la construcción de este tipo de sistemas como JAVA, XML y AJAX; el sistema se convierte en una muy buena herramienta para ir probando las ventajas de este tipo de software en el campo educativo.

Se reconoce la importancia del desarrollo de una buena interfaz gráfica de usuario que permita una sencilla interacción de los usuarios con el sistema permitiendo que los resultados del proceso de recomendación se le den a conocer de una forma clara y convincente.

\section{REFERENCIAS}

[1]. Adomavicius, G., Tuzhilin, A. (2005), Toward the next generation of recommender systems: A survey of the state-of-the-art and possible extensions. IEEE TRANSACTIONS ON KNOWLEDGE AND DATA ENGINEERING, 17:734-749.

[2]. Basu C., Hirsh H., Cohen W. (1998), Recommendation as classification: Using social and content-based information in recommendation, Proceedings of the National Conference on Artificial Intelligence, JOHN WILEY \& SONS LTD, pp. 714-720.

[3]. Burke, R. (2002), Hybrid recommender systems: Survey and experiments, User Modeling and User-Adapted Interaction, vol. 12, 2002, pp. 331-370.

[4]. Burke, Robert (2000). Knowledge-Based Recommender Systems. Encyclopedia of 
Library and Information Systems, A. Kent, ed., vol. 69, Supplement 32, Marcel Dekker.

[5]. Cerro M (2010). Grafología pedagógica. Aplicada a la orientación vocacional. NARCEA S.A de Ediciones 2010, pp. 81 84.

[6]. Housman E., Kaskela E.(1970), State of the art in selective dissemination of information, IEEE Transactions on Engineering Writing and Speech, vol. 13, 1970, pp. 78-83.

[7]. Farzan R, Brusilovsky P. (2006), Social Navigation Support in a Course.

Recommendation System. In: Proceedings of the 4th International Conference on Adaptive Hypermedia and Adaptive Web-based Systems, pp. 91100.

[8]. Goldberg D, Nichols, Oki B, Terry D. (1992). Using collaborative filtering to weave an information Tapestry. Communications of the ACM v35 n12 p61(10).

[9]. Herlocker J. (2000). Understanding and [16]. Improving Automated Collaborative Filtering Systems. University of Minnesota, Minnesota.

[10]. Pazzani M (1999). A Framework for Collaborative, Content-Based and Demographic Filtering. Artificial Intelligence Review, 13 (5-6), pp. 393408.

[11]. Pazzani M, Billsus D, (2007). ContentBased Recommendation Systems. The
Adaptive Web, Vol. 4321. Springer

Berlin/ Heidelberg. pp. 325-341.

[12]. Parameswaran A, Venetis P, GarciaMolina H (2009). Recommendation Systems with Complex Constraints: A Course Recommendation Perspective. Tech Report, Stanford Infolab, Mar 2009.

[13]. Ruiz A (2009). Estrategias de recomendación aplicadas a repositorios de recursos educativos. Tesis presentada a la Universidad Complutense de Madrid para optar por el título de Máster en Investigación en Informática.

[14]. Salamanca, P (2006). Sistema Personalizado de Reformulación Dinámica de Consultas en Exploración Web. Tesis presentada a la Universidad Nacional de Colombia para optar por el título de Magister en Ingeniería Sistemas y Computación.

[15]. Seguido, M (2009). Sistemas de recomendación para webs de información sobre la salud.

Ujjin, S; Bentley, P.J (2002). Learning User Preferences Using Evolution. In Proceedings of the 4th Asia-Pacific Conference on Simulated Evolution and Learning (Seal ‘02) 2002.

[17]. Van Setten M (2005). Supporting People In Finding Information: Hybrid Recommender Systems and Goal-Based Structuring. Tesis presentada a la Universidad de Twente para optar por un Doctorado. 